\title{
Calculation time optimization for stochastic analysis of an industrial structure
}

\author{
O. Bendaou ${ }^{1,2, a}$, A. Elhami ${ }^{2}$, A. Aannaque ${ }^{1}$ and M. Agouzoul ${ }^{1}$ \\ 1 Ecole Mohammadia d'Ingénieurs, Département Mécanique - UFR: M.C.I.C.M., BP 765, Rabat-Agdal, Morocco \\ 2 Institut National des Sciences Appliquées de Rouen, Laboratoire de Mécanique de Rouen, BP 08, avenue de l'Université, \\ 76801 Saint-Étienne du Rouvray Cedex, France
}

Received 28 December 2007, accepted 31 March 2008

\begin{abstract}
Dynamic analysis of industrial structures may be costly from a numerical point of view. Thus, the need and the importance of reducing the size of the problem become obvious. One of the main hypothesises in the use of component mode synthesis method is that the model is deterministic. That is to say the parameters used in the model have defined and fixed values. Furthermore, carrying out the structure's response involving uncertain material, geometrical parameters, limit conditions, manufacturing tolerances and/or loading conditions is essential in a global design process. Our main aim in the present work is to test and validate a method which consists in condensing the system (i.e. to reduce the number of unknowns) and carrying out the stochastic study with a noniterative method. This method makes it possible to solve these problems without using the classical method whereby a direct modal calculation combined with the Monte Carlo simulation is made. The classical method is by nature iterative and hence CPU intensive. A propeller is taken as an example in order to validate the proposed methods. The results obtained agree well with references results and show the effectiveness of the proposed method.
\end{abstract}

Key words: Modal analysis; modal synthesis; stochastic parameters; Monte Carlo simulation; Muscolino perturbation.

\section{Introduction}

The dynamic analysis of industrial mechanical systems is often costly and sometimes difficult due to the computer resources limitations. Furthermore, these mechanical systems are often constructed from several parts, which for organization reasons, are calculated and tested independently by different teams. The sub-structuring methods are often considered the only viable resolution strategy. The use of these methods is then justified by the numerical benefit and by taking organization constraints of such big projects into account. One of the most used dynamic sub-structuring strategies is based on a component mode synthesis. A literature review of these methods is synthesised in [1]. One of the pioneer works on reduction model of repetitive structures is presented in [2].

One of the main hypothesises in the study of mechanical systems is that the model is deterministic. That means, the parameters used in the model are constant. However the experimental work shows the limitations of such an assumption. This is because there are always discrepancies between what calculation and measurement results. This is due mainly to the uncertainties in geometry, material properties, limit conditions and/or loading conditions. The latter has a considerable impact on the

\footnotetext{
${ }^{a}$ Corresponding author: obendaou@gmail.com
}

vibrating behaviour of mechanical systems. This is why it is important to use numerical methods in order to take these uncertainties into count. The earlier methods were iterative [3], whereas most recent ones are noniterarative. In [4-8] many noniterative approaches are used to study mechanical systems with uncertain parameters.

\section{Modal synthesis}

We assume that a dynamical problem is divided into " $N s$ " sub-structures. In this paper, the exponents (and indices) " $s$ " will designate the number of sub-structures. Each sub-structure occupies a volume noted $\Omega^{s}$. The interface $I^{s s^{\prime}}=\Omega^{s} \cap \Omega^{s^{\prime}}$ designates the junction between the substructure " $s$ " and the sub-structure " $s$ ". $I^{s s^{\prime}}=\emptyset$ if the two domains are not in contact.

\subsection{Equations for the sub-structures}

It is assumed that each sub-structure is elastic, linear, isotropic and without any initial stress or strain. In the absence of voluminal source, the equation which models their vibratory behaviors is given by:

$$
\nabla \sigma^{s}-\rho_{s} u^{s}=0, \quad s=1, \ldots, N s
$$


$\rho_{s}, u^{s}$ and $\sigma^{s}$ are respectively the density, the displacement field and the constraints tensor of the substructure $s$.

If we note $\Gamma_{u}^{s}$ the borders of imposed displacement and $\Gamma_{f}^{s}$ the borders of imposed external force, the limit conditions associated with the substructure " $s$ " are written:

$$
\left.u^{s}\right|_{\Gamma_{u}^{s}}=\bar{u}^{s},\left.\quad \sigma^{s} n\right|_{\Gamma_{f}^{s}}=\bar{f}^{s} .
$$

In the sub-structure/sub-structure interfaces $\left(I^{s s^{\prime}}\right)$, the continuity of the displacements and the normal component of the constraints tensor must be assured. These conditions are written:

$$
\begin{aligned}
\left.\left(u^{s}-u^{s^{\prime}}\right)\right|_{I^{s s^{\prime}}} & =0, \\
\left.\left(\sigma^{s} n-\sigma^{s^{\prime}} n\right)\right|_{I^{s s^{\prime}}} & =0 .
\end{aligned}
$$

\subsection{Variational formulations}

We assume that $u^{s *}$ is a virtual field associated with the substructure " $s$ ". The equation (1) is integrated over the domain $\Omega^{s}$. After integration by parts and then application of the conditions (2), (3) and (4), the variational problem consists in finding $u^{s}$ such that $\left\{\left.u^{s}\right|_{\Gamma_{u}^{s}}=\bar{u}^{s}\right\}$ :

$$
\begin{aligned}
& \int_{\Omega^{s}} \rho_{s} u^{s *} \ddot{u}^{s} d V+\int_{\Omega^{s}} \varepsilon^{s *}: \sigma^{s} d V=\int_{\Gamma_{f}^{s}} u^{s *} \bar{f}^{s} d S \\
&+\sum_{\substack{s^{\prime}=1 \\
s^{\prime} \neq s}}^{N s} \int_{I^{s s^{\prime}}} u^{s *}\left(\sigma^{s^{\prime}} n\right) d S \\
& \forall u^{s *} /\left\{\left.u^{s *}\right|_{\Gamma_{u}^{s}}=0\right\} \quad s=1, \ldots, N s
\end{aligned}
$$

where $\varepsilon$ is the deformations tensor and $n$ the outgoing normal from $\Omega^{s}$.

\subsection{Discretization by finite elements}

The discretization of the structural variational problems (5) by finite elements and the application of the Galerkin method lead to the following algebraic form:

$$
\left[\mathbf{M}^{s}\right]\left\{\ddot{\mathbf{u}}^{s}\right\}+\left[\mathbf{K}^{s}\right]\left\{\mathbf{u}^{s}\right\}=\left\{\mathbf{f}^{s}\right\}+\sum_{\substack{s^{\prime}=1 \\ s^{\prime} \neq s}}^{N s}\left\{\mathbf{f}_{I}^{s s^{\prime}}\right\} s=1, \ldots, N s .
$$

The vector $\left\{\mathbf{u}^{s}\right\}$ contains all the unknown degrees of freedom associated with the structural displacements of the sub-structure " $s$ " (the known degrees of freedom on the border $\Gamma_{u}^{s}$, are not included in this vector). $\left[\mathbf{M}^{s}\right],\left[\mathbf{K}^{s}\right]$ and $\left\{\mathbf{f}^{s}\right\}$ are respectively the mass matrix of the substructure " $s$ ", its stiffness matrix and the vector of the external equivalent forces (including the inherent excitations of imposed displacements on the border $\Gamma_{u}^{s}$ ).
The term $\left\{\mathbf{f}_{I}^{s s^{\prime}}\right\}$ represents physically the inter-facial forces applied on the sub-structure " $s$ " by the adjacent sub-structure " $s$ ".

We assemble the "Ns" substructures according to a total vector containing the all degrees of freedom, organized according to the following form:

$$
\langle\mathbf{u}\rangle=\left\langle\mathbf{u}^{1} \mathbf{u}^{2} \ldots \mathbf{u}^{N s}\right\rangle .
$$

The assembly of the formulation (6) leads to the following algebraic system:

$$
[\mathbf{M}]\{\ddot{\mathbf{u}}\}+[\mathbf{K}]\{\mathbf{u}\}=\{\mathbf{f}\}+\left\{\mathbf{f}_{I}\right\}
$$

where:

$$
\begin{gathered}
{[\mathbf{M}]=\left[\begin{array}{lll}
\mathbf{M}^{1} & & \\
& \ddots & \\
& & \mathbf{M}^{N s}
\end{array}\right], \quad[\mathbf{K}]=\left[\begin{array}{lll}
\mathbf{K}^{1} & & \\
& \ddots & \\
& & \mathbf{K}^{N s}
\end{array}\right],} \\
\{\mathbf{f}\}=\left\{\begin{array}{l}
\mathbf{f}^{1} \\
\vdots \\
\mathbf{f}^{N s}
\end{array}\right\}, \quad\left\{\mathbf{f}_{I}\right\}=\left\{\begin{array}{l}
\sum_{s^{\prime} \neq 1} \mathbf{f}_{I}^{1 s^{\prime}} \\
\vdots \\
\sum_{s^{\prime} \neq N s} \mathbf{f}_{I}^{N s s^{\prime}}
\end{array}\right\} .
\end{gathered}
$$

\subsection{Calculation of local modes}

The degrees of freedom vector of each sub-structure " $s$ " is partitioned into two groups: the internal degrees freedom (index $i$ ) and those at the junctions (index $j$ ). The latter correspond to the degrees of freedom located on the interface between the sub-structure " $s$ " and the all other adjacent sub-structures. Then, we write:

$$
\begin{aligned}
& \left\langle\mathbf{u}^{s}\right\rangle=\left\langle\mathbf{u}_{i}^{s} \mid \mathbf{u}_{j}^{s}\right\rangle, \quad\left[\mathbf{M}^{s}\right]=\left[\begin{array}{cc}
\mathbf{M}_{i i}^{s} & \mathbf{M}_{i j}^{s} \\
\mathbf{M}_{j i}^{s} & \mathbf{M}_{j j}^{s}
\end{array}\right], \\
& {\left[\mathbf{K}^{s}\right]=\left[\begin{array}{ll}
\mathbf{K}_{i i}^{s} & \mathbf{K}_{i j}^{s} \\
\mathbf{K}_{j i}^{s} & \mathbf{K}_{j j}^{s}
\end{array}\right] .}
\end{aligned}
$$

According to the Craig and Bampton method, the chosen local modes correspond to the modes with fixed interfaces $I^{s s^{\prime}}$. They verify the following eigenvalue problem:

$$
\left[\mathbf{K}_{i i}^{s}-\omega^{2} \mathbf{M}_{i i}^{s}\right]\left\{\psi^{\mathrm{s}}\right\}=\{0\}, \quad s=1, \ldots, N s .
$$

These orthogonal modes are enriched by static modes of connection. The latter are defined as being the static deformation of the considered sub-structure, when a unit displacement is applied by turns to each of its junction degrees of freedom, the others being forced to be 0 .

The local modal base of a sub-structure " $s$ " is therefore given by:

$$
\left[\boldsymbol{\varphi}^{s}\right]=\left[\begin{array}{ll}
\boldsymbol{\psi}^{s}-\mathbf{K}_{i i}^{s-1} \mathbf{K}_{i j}^{s} \\
0 & \mathbf{I}_{j j}^{s}
\end{array}\right]
$$


where $\left[\boldsymbol{\psi}^{s}\right]$ represents the matrix of the modes with fixed interfaces retained after truncation and which are disposed in columns. The strategy of choice of the retained number of modes for each sub-structure consists in retaining all modes contained in the multiple band of the useful frequency $(F u)$, that is to say twice the useful band $([002 F u])[9]$.

The physical degrees of freedom of each substructure can then be decomposed on their respective local modal base:

$$
\left\{\mathbf{u}^{s}\right\}=\left[\boldsymbol{\varphi}^{s}\right]\left\{\boldsymbol{\alpha}^{s}\right\}, \quad s=1, \ldots, N s .
$$

$\left\{\boldsymbol{\alpha}^{s}\right\}$ is the vector of the generalized coordinates associated with the sub-structure " $s$ ", containing:

- the coefficients associated to the fixed interfaces modes;

- the junctions physical degrees of freedom.

\subsection{Model reduction}

The local decompositions (14) can be assembled, as follows:

$$
\{\mathbf{u}\}=[\varphi]\{\boldsymbol{\alpha}\}
$$

where:

$$
[\boldsymbol{\varphi}]=\left[\begin{array}{ccc}
\boldsymbol{\varphi}^{1} & & 0 \\
& \ddots & \\
0 & & \boldsymbol{\varphi}^{N s}
\end{array}\right] \text { and }\{\boldsymbol{\alpha}\}=\left\{\begin{array}{l}
\boldsymbol{\alpha}^{1} \\
\vdots \\
\boldsymbol{\alpha}^{N s}
\end{array}\right\}
$$

Then, equation (8) becomes after projection:

$$
\left[\mathbf{M}_{\alpha}\right]\{\ddot{\boldsymbol{\alpha}}\}+\left[K_{\alpha}\right]\{\boldsymbol{\alpha}\}=\left\{\mathbf{f}_{\alpha}\right\}+[\boldsymbol{\varphi}]^{t}\left\{\mathbf{f}_{I}\right\}
$$

where:

$$
\left[\mathbf{M}_{\alpha}\right]=[\boldsymbol{\varphi}]^{t}[\mathbf{M}][\boldsymbol{\varphi}], \quad\left[\mathbf{K}_{\alpha}\right]=[\boldsymbol{\varphi}]^{t}[\mathbf{K}][\boldsymbol{\varphi}],\left[\mathbf{f}_{\alpha}\right]=[\boldsymbol{\varphi}]^{t}[\mathbf{f}]
$$

Now, it is necessary to take the continuity conditions at the structure/structure interfaces into account. Indeed, the degrees of freedom of $\{\boldsymbol{\alpha}\}$ are not linearly independent. The linear relations between these degrees of freedom result from the equality of displacements at the structure/structure interfaces. They can be expressed by a connectivity global matrix $[\mathbf{S}]$ :

$$
\{\boldsymbol{\alpha}\}=[\mathbf{S}]\{\mathbf{q}\}
$$

where $\{\mathbf{q}\}$ contains only the linearly independent degrees of freedom. [S] characterizes the inter sub-structures connectivity. If we use the Craig and Bampton method, $[\mathbf{S}]$ is Boolean and easy to express since the junction physical degrees of freedom belong explicitly to the generalized unknown factors $\{\boldsymbol{\alpha}\}$.

According to the conditions (4), the compatibility equations are as follows:

$$
\left\{\mathbf{f}^{s s^{\prime}}\right\}+\left\{\mathbf{f}^{s^{\prime} s}\right\}=\{0\} .
$$

Then, we can show that these equations imply:

$$
[\mathbf{S}]^{t}[\varphi]^{t}\left\{\mathbf{f}_{I}\right\}=[\mathbf{S}]^{t}\left\{\mathbf{f}_{I}\right\}=0
$$

Thus, the final system to be solves is written:

$$
\left[\mathbf{M}_{q}\right]\{\ddot{\mathbf{q}}\}+\left[\mathbf{K}_{q}\right]\{\mathbf{q}\}=\left\{\mathbf{f}_{q}\right\}
$$

with:

$$
\begin{aligned}
{\left[\mathbf{M}_{q}\right] } & =[\mathbf{S}]^{t}\left[\mathbf{M}_{\alpha}\right][\mathbf{S}], \\
{\left[\mathbf{K}_{q}\right] } & =[\mathbf{S}]^{t}\left[\mathbf{K}_{\alpha}\right][\mathbf{S}], \\
{\left[\mathbf{f}_{q}\right] } & =[\mathbf{S}]^{t}\left[\mathbf{f}_{\alpha}\right] .
\end{aligned}
$$

Compared with the system (8), this model is considerably reduced since its size corresponds to the total number of orthogonal local modes retained after truncation, to which it is added the total number of junction degrees of freedom.

\section{Stochastic study}

\subsection{Monte Carlo simulation}

The estimation of the natural frequency moments (average and variance) of a structure could be obtained by the Monte Carlo simulation [3]. This is a very widely used method despite its high CPU cost, and it is used as a reference for others calculation methods. The natural frequency $\lambda$ is seen as a random variable image of the basic random variables $\left\{\alpha_{p}\right\}_{(p=1, \ldots \ldots, P)}$. The simulation is carried out by constructing a sample $\left((\lambda)_{1},(\lambda)_{2}, \ldots,(\lambda)_{n}\right)$ of the random variable $\lambda$ and to treat this sample by the usual statistics techniques. The " $n$ " simulations are done in an independent way according to the distribution law of the random vector.

The average of $\lambda$ is given by:

$$
E[\lambda]=\frac{1}{n} \sum_{i=1}^{n}(\lambda)_{i} .
$$

The variance of $\lambda$ is given by:

$$
\operatorname{Var}[\lambda]=\frac{1}{n-1} \sum_{i=1}^{n}\left[(\lambda)_{i}-E(\lambda)\right]^{2}
$$

The accuracy of the results does not depend on the number of variables considered.

\subsection{Perturbation methods}

The perturbation methods are very widely used in the stochastic finite elements domain. They are based on a development in Taylor series of the natural frequencies (respectively the F.R.F. or the dynamical response) in relation to the basis random physical variables, mechanical properties, geometrical characteristics or applied forces. 
The perturbation methods calculate the average and the variance of the natural frequencies of a mechanical structure that has uncertain parameters. This method is used in many areas in order to solve linear and non-linear problems, for either static or dynamic cases.

The Muscolino perturbation method [6] could be used for a mechanical system where the random parameters are independent. It is based on a development into a firstorder Taylor series.

For a structure with uncertain parameters, it is assumed that the mass matrix and the stiffness matrix are functions of the random variables $\left\{\alpha_{p}\right\}_{(p=1, \ldots \ldots, P)}$. And we note $\lambda_{i}$ the $i$ th natural frequency.

The vector of the average parameters is defined by $\{\bar{\alpha}\}$, and the quantity $d \alpha$ is defined by

$$
\{d \alpha\}=\{\alpha\}-\{\bar{\alpha}\} \text {. }
$$

The following notation is used to simplify the writing:

$$
[\mathbf{A}]^{0}=\left.[\mathbf{A}]\right|_{\{\bar{\alpha}\}}, \quad[\mathbf{A}]^{p}=\left.\frac{\partial[\mathbf{A}]}{\partial \alpha_{p}}\right|_{\{\bar{\alpha}\}}
$$

$[\mathbf{A}]^{0}$ and $[\mathbf{A}]^{p}$ are deterministic. The repetition of the indice " $p$ " two times implies a summation.

For a Muscolino perturbation method, we have:

$$
\begin{gathered}
{[\mathbf{K}]=[\mathbf{K}]^{0}+[\mathbf{K}]^{p}\left\{d \alpha_{p}\right\}} \\
{[\mathbf{M}]=[\mathbf{M}]^{0}+[\mathbf{M}]^{p}\left\{d \alpha_{p}\right\}} \\
\{\mathbf{f}\}=\{\mathbf{f}\}^{0}+\{\mathbf{f}\}^{p}\left\{d \alpha_{p}\right\} .
\end{gathered}
$$

The eigenvalues-eigenvectors equation of "0" order are:

$$
\left([\mathbf{K}]^{0}-\left(\lambda_{i}\right)^{0}[\mathbf{M}]^{0}\right)\left\{\boldsymbol{\psi}_{i}\right\}^{0}=0
$$

The eigenvalues-eigenvectors equation of "1" order gives are:

$$
\left(\lambda_{i}\right)^{p}=\frac{{ }^{t}\left\{\boldsymbol{\psi}_{i}\right\}^{0}\left([\mathbf{K}]^{p}-\left(\lambda_{i}\right)^{0}[\mathbf{M}]^{p}\right)\left\{\boldsymbol{\psi}_{i}\right\}^{0}}{{ }^{t}\left\{\boldsymbol{\psi}_{i}\right\}^{0}\left([\mathbf{M}]^{0}\right)\left\{\boldsymbol{\psi}_{i}\right\}^{0}}
$$

The average is given by:

$$
E\left[\lambda_{i}\right]=\left(\lambda_{i}\right)^{0}
$$

The variance is given by:

$$
\operatorname{Var}\left[\lambda_{i}\right]=\left(\left(\lambda_{i}\right)^{p}\right)^{2} \operatorname{Var}\left(\alpha_{p}\right) .
$$

The Muscolino method has the advantage of requiring less calculation than the one based on the development into a second-order Taylor series.
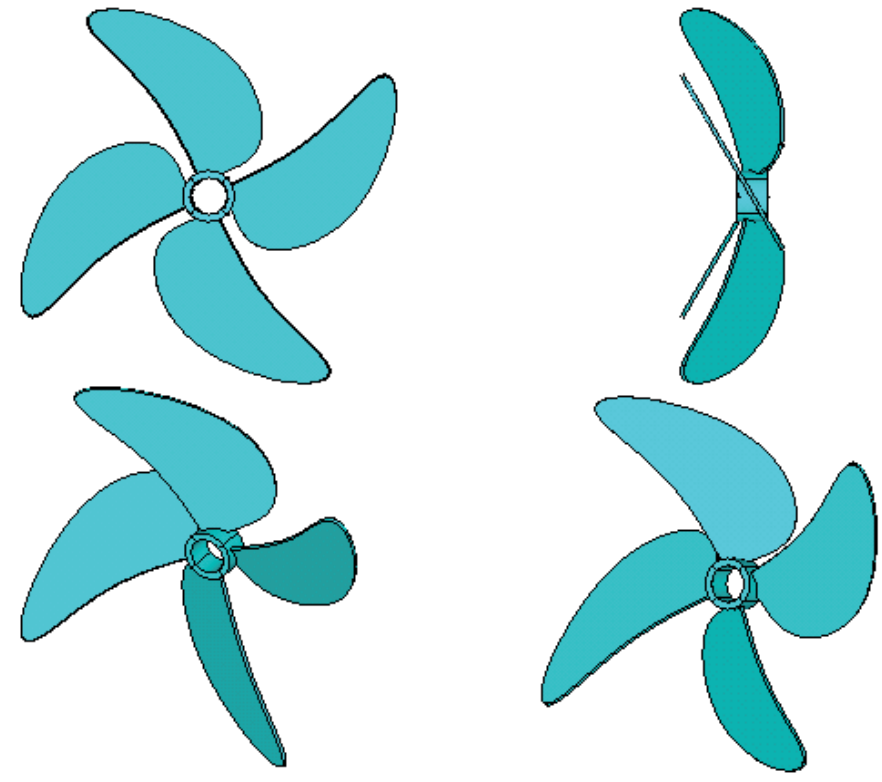

Fig. 1. Boat propeller.

\subsection{Perturbation methods with modal synthesis}

For a mechanical system whose dofs are reduced by the modal synthesis method, it is assumed that the modal bases are deterministic for the Muscolino perturbation method. This assumption is justified, since the perturbation method is only applied to systems with weakly varying parameters. Therefore:

$$
\begin{aligned}
& {\left[\mathbf{M}_{q}\right]^{0}=[\mathbf{S}]^{t}[\varphi]^{t}[\mathbf{M}]^{0}[\varphi][\mathbf{S}]} \\
& {\left[\mathbf{M}_{q}\right]^{p}=[\mathbf{S}]^{t}[\varphi]^{t}[\mathbf{M}]^{p}[\varphi][\mathbf{S}]}
\end{aligned}
$$

$$
\begin{aligned}
& {\left[\mathbf{K}_{q}\right]^{0}=[\mathbf{S}]^{t}[\varphi]^{t}[\mathbf{K}]^{0}[\varphi][\mathbf{S}],} \\
& {\left[\mathbf{K}_{q}\right]^{p}=[\mathbf{S}]^{t}[\varphi]^{t}[\mathbf{K}]^{p}[\varphi][\mathbf{S}] .}
\end{aligned}
$$

\section{Industrial application}

In order to validate the suggested methods, we studied the dynamic behavior of a boat propeller. The geometrical model of this propeller (Fig. 1) was designed using "SOLIDWORKS" and "ANSYS". The mesh as well as the geometrical sub-structuring (Fig. 2) was carried out with "ANSYS". Whereas the processor part was carried out on the one hand with "ANSYS" for deterministic calculation without dofs reduction (the result of this calculation will be regarded as a reference), and on the other hand with codes elaborated with "MATLAB" (on the basis of the mesh carried out with "ANSYS") for deterministic and stochastic analysis, with and without dofs reduction.

The mesh was made with tetrahedral elements. For calculation with modal synthesis (dofs reduction) we divided the propeller into four sub-structures. 
Table 1. The six first natural frequencies of the propeller (and the relative errors) $(\mathrm{Hz})$.

\begin{tabular}{ccccc}
\hline \multicolumn{2}{c}{ Direct calculation } & $\begin{array}{c}\text { Calculation with modal } \\
\text { synthesis }\end{array}$ \\
\hline mode & $\begin{array}{c}\text { with ANSYS } \\
\text { (reference) }\end{array}$ & with MATLAB & with MATLAB \\
\hline 1 & 100 & $100(0.0 \%)$ & $100(0.0 \%)$ \\
2 & 295 & $295(0.0 \%)$ & $295(0.0 \%)$ \\
3 & 346 & $346(0.0 \%)$ & $346(0.0 \%)$ \\
4 & 915 & $914(0.1 \%)$ & $914(0.1 \%)$ \\
5 & 1225 & $1224(0.1 \%)$ & $1224(0.1 \%)$ \\
6 & 1519 & $1518(0.1 \%)$ & $1518(0.1 \%)$ \\
\hline Dofs & \multicolumn{3}{c}{2286} & \multicolumn{2}{c}{96} \\
number & \multicolumn{3}{c}{} \\
\hline
\end{tabular}
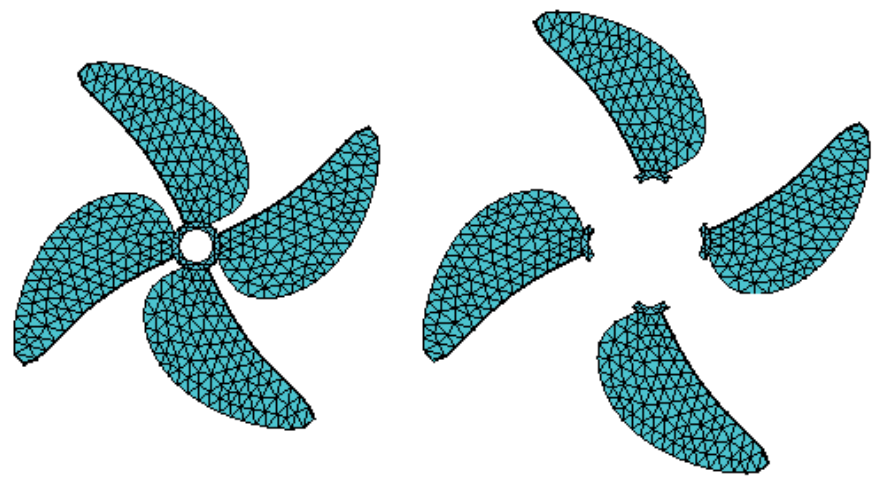

Fig. 2. Mesh and sub-structuring of the boat propeller.

\subsection{Deterministic modal analysis}

In Table 1, the modal analysis of the propeller is presented, and its six first natural frequencies calculed with:

- "ANSYS" without dofs reduction (reference);

- "MATLAB" without dofs reduction;

- "MATLAB" with dofs reduction (modal synthesis).

are compared. The calculations are made by assuming that the material parameters are fixed:

$$
E=2.1 e^{11}[\mathrm{~Pa}], \quad \rho=7860\left[\mathrm{Kg} / \mathrm{m}^{3}\right] \text { and } \nu=0.3 .
$$

The time that calculation with "MATLAB" required:

- for direct calculation: 6 min $30 \mathrm{~s}$;

- for calculation with modal synthesis: $62 \mathrm{~ms}$.

This result clearly shows the substantial time CPU saving made using the model reduction by modal synthesis.

\subsection{Stochastic modal analysis}

After the deterministic modal analysis, we investigate the modal analysis in a case where the Young modulus and the density of the propeller are random variables with normal distribution and for which we know the averages and the standard deviations (Tab. 2).

Computer codes were elaborate using "MATLAB" to calculate the moments (averages and standard deviations) of the first 6 natural frequencies of the propeller, with 3 different methods:
Table 2. Random variables and statistics parameters of the propeller.

\begin{tabular}{cccc}
\hline & Distribution & Mean & Standard deviation \\
\hline$E(\mathrm{~Pa}) \times \mathrm{e}^{11}$ & normal & 2.1 & 0.021 \\
$\rho\left(\mathrm{Kg} / \mathrm{m}^{3}\right)$ & normal & 7860 & 78.60 \\
\hline
\end{tabular}

- method 1 (classical method): direct calculation with Monte Carlo simulation (for 100 samples);

- method 2: direct calculation with Muscolino perturbation method;

- method 3 (proposed method): modal synthesis with Muscolino perturbation method.

Let's note that "method 1" is a classical method which we will be taken as a reference.

The result of this study is presented in Table 3 (means) and Table 4 (standard deviations).

The time required to obtain the 6 first natural frequencies moments of the propeller using "MATLAB":

- method 1: 17 h 36 min;

- method 2: $26 \min 30 \mathrm{~s}$;

- method 3: $234 \mathrm{~ms}$.

The propeller example shows that the CPU time saving is very important; when the dynamical study of a structure with random parameters is carried out by the modal synthesis method allied to the Muscolino perturbation method.

\section{Conclusion}

In order to be able to solve large dynamical problems with reasonable computing time, we proposed to use the modal reduction method combined with the Muscolino Perturbation method. The obtained results in the case of a propeller show the validity and the potentialities of the proposed method. In the deterministic case, the results obtained well with the references results. Furthermore, results obtained using dofs reduction agree well with the ones without dofs reduction. As further example, a propeller with random Young modulus and its density was considered. The obtained moments (averages and 
Table 3. The Means of the six first natural frequencies of the propeller (and the relative errors) $(\mathrm{Hz})$.

\begin{tabular}{ccccc}
\hline \multicolumn{4}{c}{ Means } \\
\hline mode & $\begin{array}{c}\text { method 1 } \\
\text { - classical method - }\end{array}$ & method 2 & $\begin{array}{c}\text { method 3 } \\
\text { - proposed method - }\end{array}$ \\
\hline 1 & 100 & $100(0.0 \%)$ & $100(0.0 \%)$ \\
2 & 295 & $295(0.0 \%)$ & $295(0.0 \%)$ \\
3 & 346 & $346(0.0 \%)$ & $346(0.0 \%)$ \\
4 & 914 & $914(0.0 \%)$ & $914(0.0 \%)$ \\
5 & 1224 & $1224(0.0 \%)$ & $1224(0.0 \%)$ \\
6 & 1519 & $1518(0.1 \%)$ & $1518(0.1 \%)$ \\
\hline
\end{tabular}

Table 4. The Standard Deviations of the six first natural frequencies of the propeller (and the relative errors) (Hz).

\begin{tabular}{cccc}
\hline \multicolumn{3}{c}{ Standard deviations } \\
\hline mode & $\begin{array}{c}\text { method 1 } \\
\text { - classical method - }\end{array}$ & method 2 & $\begin{array}{c}\text { method 3 } \\
\text { - proposed method - }\end{array}$ \\
\hline 1 & 12 & $12(0.0 \%)$ & $12(0.0 \%)$ \\
2 & 35 & $35(0.0 \%)$ & $35(0.0 \%)$ \\
3 & 41 & $41(0.0 \%)$ & $41(0.0 \%)$ \\
4 & 109 & $109(0.0 \%)$ & $109(0.0 \%)$ \\
5 & 146 & $145(0.7 \%)$ & $145(0.7 \%)$ \\
6 & 181 & $180(0.5 \%)$ & $180(0.5 \%)$ \\
\hline
\end{tabular}

standard deviations) of the propeller natural frequencies calculated with Muscolino methode (with and without modal reduction) were compared to the Monte Carlo simulation without dofs reduction (classical method). The obtained results using the three methods do agree, with a large computing time saving for the proposed method.

\section{References}

1. R.R. Craig. ASME J. Vib. Acoust. 117, 207 (1995)

2. A. El Hami, B. Radi. Comput. Struct. 58, 973 (1996)

3. M. Shinozuka. International Journal Computer Structural 2, 855 (1972)
4. N. Impollonia, G. Ricciardi. Prob. Eng. Mech. 21, 171 (2005)

5. M. Kleiber, T.D. Hien, The stochastic finite element method (New York, John Wiley, 1992)

6. G. Muscolino, G. Ricciardi, N. Impollonia. Prob. Eng. Mech. 15, 199 (1999)

7. B. Van den Nieuwenhof, J.P. Coyette. Computers Methods in Applied Mechanics and Engineering 192, 3705 (2003)

8. O. Dessombz, A. Diniz, F. Thouverez, L. Jézéquel, Analysis of stochastic structures: Pertubation method and projection on homogeneous chaos, In Proceedings of the 17th Int. Modal Analysis Conference IMAC-SEM (KissimmeeFloride, Feb, 1999), pp. 1763-1777

9. A. El Hami, G. Lallement, P. Minotti, H. Belhadj, S. Cogan. Comput. Struct. 48, 975 (1993) 